\title{
Capacity Bounds for Amplitude-Constrained AWGN MIMO Channels with Fading
}

\author{
Antonino Favano* ${ }^{*}$, Marco Ferrari ${ }^{\dagger}$, Maurizio Magarini*, and Luca Barletta* \\ *Politecnico di Milano, Milano, Italy, \{antonino.favano, maurizio.magarini, luca.barletta\}@polimi.it \\ ${ }^{\dagger}$ CNR-IEIIT, Milano, Italy, marco.ferrari@ieiit.cnr.it
}

\begin{abstract}
We evaluate capacity bounds for multiple-input multiple-output (MIMO) additive white Gaussian noise (AWGN) fading channels subject to input amplitude constraints. We focus on two practical cases, in which the transmitter: (i) employs a single antenna amplifier, which induces a constraint on the norm of the input vector, and (ii) it employs multiple amplifiers, one per antenna, which leads to independent constraints on the amplitude of each input vector entry. For both cases, we evaluate the asymptotic capacity gap between upper and lower bounds at high signal-to-noise ratio.
\end{abstract}

\section{INTRODUCTION}

The information capacity of a channel subject to amplitudeconstrained input is of great interest for its practical applications. The case of scalar Gaussian channels is discussed in [1] and extended to quadrature Gaussian channels in [2]. Moreover, multiple-input multiple-output (MIMO) Gaussian channels are investigated in [3]. In [4] McKellips presents a tight capacity upper bound for the scalar case with closed-form expression. In [5] the McKellips' bound is rederived, refined, and extended to complex scalar and MIMO additive white Gaussian noise (AWGN) channels without fading. Both [3] and [5] focus on total amplitude constraints, which limit the norm of the input vector. In [6], it is discussed the capacity of MIMO AWGN channels subject to per-antenna constraints, which limit the amplitude of each entry of the input vector independently. Finally, in [7] the authors derive bounds for MIMO AWGN fading channels with full channel state information and arbitrary amplitude constraints on the input, with particular emphasis on both per-antenna and total amplitude constraints.

\section{Contribution}

In this paper, we extend the McKellips-Type upper bound of [5] to complex MIMO AWGN channels with fading. In addition to the case of total amplitude constraints, we adapt the McKellips-Type bound to systems with per-antenna input constraints where the right singular vectors of the channel matrix form the standard basis. The investigated constraints refer to implementations of practical interest. In the case of total amplitude constraints, all transmitting antennas employ concurrently the same amplifier, while for the per-antenna constraints, the transmitter relies on multiple amplifiers, one for each antenna [8]. Moreover, we propose a refinement of the entropy power inequality (EPI) lower bound in [7], improving its tightness at low-to-intermediate signal-to-noise ratio (SNR), especially in presence of strong fading attenuation. Finally, for both cases, we compute the asymptotic gap between the derived upper and lower capacity bounds at high SNR.

The paper is organized as follows: in Sec. II, we describe the MIMO system model. In Sec. III, we provide capacity upper and lower bounds for the per-antenna amplitude constraint. We also discuss the details about the EPI lower bound refinement, and then we evaluate the gap between upper and lower bounds at high SNR. In Sec. IV, we perform a similar capacity analysis for the total amplitude constraint, and we compare our results to the state of the art. Section V concludes the paper.

\section{Notation}

We use bold letters for vectors ( $\mathbf{x}$ ), uppercase letters for random variables $(X)$, calligraphic uppercase letters for the support of a random vector distribution $(\mathcal{X})$, and uppercase sans serif letters for matrices $(H)$. We denote by $|\mathrm{H}|, \mathrm{H}^{T}$, and $\mathrm{H}^{H}$ the determinant, the transposed, and the Hermitian transposed of a matrix $\mathrm{H}$, respectively. We define $\mathbf{X}_{k}^{j} \triangleq$ $\left(X_{k}, X_{k+1}, \ldots, X_{j}\right)^{T}$ with $k \leq j$ and $\mathrm{H} \mathcal{X} \triangleq\{\mathbf{y}: \mathbf{y}=$ $\mathrm{Hx}, \mathbf{x} \in \mathcal{X}\}$. We represent the $k \times 1$ vector of zeros by $\mathbf{0}_{k}$ and the $k \times k$ identity matrix by $\mathrm{I}_{k}$. Finally, $\mathcal{C N}(\boldsymbol{\mu}, \Sigma)$ denotes a complex multivariate Gaussian distribution with mean vector $\boldsymbol{\mu}$ and covariance matrix $\Sigma$.

\section{SySTEM MODEL}

The considered MIMO AWGN fading channel is described by the following input-output relationship

$$
\widetilde{\mathbf{Y}}=\widetilde{\mathrm{H}} \cdot \widetilde{\mathbf{X}}+\widetilde{\mathbf{Z}}
$$

where $\widetilde{\mathbf{Z}}$ is the noise vector with distribution $\mathcal{C N}\left(\mathbf{0}_{N_{\mathrm{R}}}, 2 \mathrm{I}_{N_{\mathrm{R}}}\right)$ and $\widetilde{\mathrm{H}}$ is any $N_{\mathrm{R}} \times N_{\mathrm{T}}$ full rank channel fading matrix known both at the transmitter and at the receiver. Using the singular value decomposition (SVD) for $\widetilde{H}=U \widetilde{\Lambda} V^{H}$, we can define the following model

$$
\begin{aligned}
\mathbf{Y}=\mathrm{U}^{H} \tilde{\mathbf{Y}} & =\mathrm{U}^{H}\left(\mathrm{U} \frac{\tilde{\Lambda}}{\lambda_{\max }} \lambda_{\max } \mathrm{V}^{H} \cdot \widetilde{\mathbf{X}}+\widetilde{\mathbf{Z}}\right) \\
& =\Lambda \cdot \mathbf{X}+\mathbf{Z},
\end{aligned}
$$

where the noise vector $\mathbf{Z}$ is statistically equivalent to $\widetilde{\mathbf{Z}}, \lambda_{\max }$ is the largest element of $\widetilde{\Lambda}, \Lambda=\widetilde{\Lambda} / \lambda_{\max }$, and $\mathbf{X}=\lambda_{\max } \mathrm{V}^{H} \widetilde{\mathbf{X}}$. The support of the input $\widetilde{\mathbf{X}}$ is denoted by $\widetilde{\mathcal{X}} \subseteq \mathbb{C}^{N_{\mathrm{T}}}$, and for $\mathbf{X}$ in (3), we have $\mathcal{X}=\lambda_{\max } \mathrm{V}^{H} \widetilde{\mathcal{X}}$. From here on, we consider $N_{\mathrm{R}}=N_{\mathrm{T}}=N$. We define $\lambda_{k}$ as the element $(k, k)$ of the 


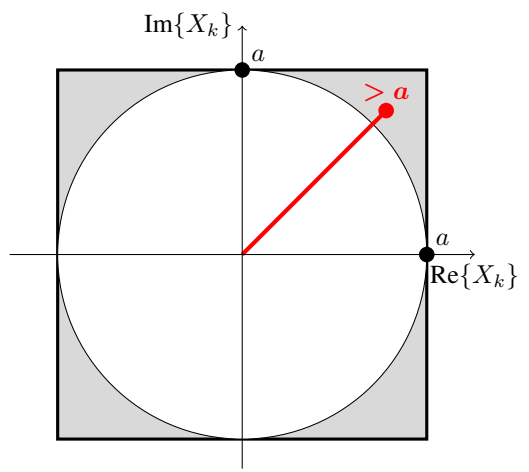

Figure 1. Resulting constraint region for the complex input (circle) and for the real and imaginary part of the signal, independently (square).

matrix $\Lambda$, and we let $\boldsymbol{\lambda}=\left(\lambda_{1}, \ldots, \lambda_{N}\right)^{T}$ be sorted such that $\lambda_{1} \geq \cdots \geq \lambda_{N}$. Finally, the MIMO channel capacity is

$$
\begin{aligned}
C_{\text {MIMO }} & =\max _{F_{\mathbf{X}}: \operatorname{supp}\left(F_{\mathbf{X}}\right) \subseteq \mathcal{X}} I(\mathbf{X} ; \mathbf{Y}) \\
& =\max _{F_{\wedge \mathbf{X}}: \operatorname{supp}\left(F_{\wedge \mathbf{X}}\right) \subseteq \wedge \mathcal{X}} I(\Lambda \mathbf{X} ; \mathbf{Y}),
\end{aligned}
$$

where $F_{\mathbf{X}}$ is the input probability distribution function. In the following sections, we focus on two specific input constraints. For each of them, we characterize the support of the input distribution $\mathcal{X}$ and the SNR definition in their relative section.

\section{Per-Antenna Amplitude Constraint}

Let the complex input vector be subject to a per-antenna amplitude constraint, which in model (1) is given by $\widetilde{\mathbf{X}} \in$ $\widetilde{\mathcal{X}}=\operatorname{Box}(\widetilde{\mathbf{a}}) \triangleq\left\{\widetilde{\mathbf{x}}:\left|\widetilde{x}_{k}\right| \leq \widetilde{a}_{k}, k=1, \ldots, N\right\}$, with $\widetilde{\mathbf{a}}=\left(\widetilde{a}_{1}, \ldots, \widetilde{a}_{N}\right) \in \mathbb{R}_{+}^{N}$ being the set of amplitude constraints. In order to bring this constraint to model (3), we focus the analysis on systems with channel matrices $\widetilde{\mathrm{H}}=U \widetilde{\Lambda} \mathrm{V}^{H}$ whose right singular vectors form the standard basis, i.e., $\mathrm{V}^{H}=\mathrm{I}_{N}$. Therefore, the per-antenna constraint for model (3) becomes $\mathbf{X} \in \mathcal{X}=\operatorname{Box}(\mathbf{a})$, with $\mathbf{a}=\widetilde{\mathbf{a}} \lambda_{\max }$ and $a_{k}=\widetilde{a}_{k} \lambda_{\max }, \forall k$. Moreover, since unequal amplitude constraints can be compensated for in the fading matrix, we can consider $a_{k}=a, \forall k$ without loss of generality. The amplitude constraint on the complex input $\left|X_{k}\right|<a$ can also be expressed as $X_{k} \in \mathcal{X}_{k}=\mathcal{B}_{2}(a) \triangleq\left\{x_{k}:\left|x_{k}\right| \leq a\right\}$. We remark that under a per-antenna amplitude constraint, real-valued vector models and complex-valued vector models are not equivalent, leading to different capacity results. Specifically, vectorizing an $N$-dimensional complex model into a $2 N$-dimensional real model, like in [7], and then applying an amplitude constraint to each real dimension independently, increases the capacity. This occurs because the constraint on the vectorized complex input $X_{k}$ is weaker than $\left|X_{k}\right| \leq a$, as shown in Fig. 1. Out of the two, we think that the complex-valued model correctly interprets the technological constraint imposed by the amplifiers. Finally, since the entries of $\mathbf{Z}$ are independent and with variance equal to 2 , given the constraint $a$ we define the SNR as $a^{2} /(2 N)$.

\section{A. Upper Bound}

Let $C_{k}$ be the capacity of the $k$ th subchannel. We have

$$
\begin{aligned}
& C_{\text {MIMO }} \leq \max _{F_{\wedge \mathrm{X}}: \operatorname{supp}\left(F_{\wedge \mathrm{X}}\right) \subseteq \wedge \mathcal{X}} \sum_{k=1}^{N} I\left(\lambda_{k} X_{k} ; Y_{k}\right) \\
& \leq \sum_{k=1}^{N} \max _{F_{\lambda_{k} X_{k}}: \operatorname{supp}\left(F_{\lambda_{k} X_{k}}\right) \subseteq \lambda_{k} \mathcal{X}_{k}} I\left(\lambda_{k} X_{k} ; Y_{k}\right)=\sum_{k=1}^{N} C_{k} .
\end{aligned}
$$

The first inequality follows from $\mathbf{Z}$ being isotropic. The second inequality holds by swapping the order of the maximization and of the sum. Notice that, for the special case of per-antenna (PA) constraints with $\mathrm{V}^{H}=\mathrm{I}_{N}$, it holds $\lambda_{1} \mathcal{X}_{1} \times \cdots \times \lambda_{N} \mathcal{X}_{N}=\wedge \mathcal{X}$. The McKellips-Type bound of [5] for the $k$ th subchannel, $\bar{C}_{k, \mathrm{PA}}$, reads as follows

$$
C_{k} \leq \bar{C}_{k, \mathrm{PA}}=\log \left(1+\lambda_{k} a \sqrt{\pi / 2}+\frac{\lambda_{k}^{2} a^{2}}{2 e}\right) .
$$

Finally, by plugging (8) into (7), we define $\bar{C}_{\mathrm{PA}}$ as the capacity upper bound for the MIMO channel

$$
C_{\mathrm{MIMO}} \leq \bar{C}_{\mathrm{PA}}=\sum_{k=1}^{N} \bar{C}_{k, \mathrm{PA}} .
$$

\section{B. Lower Bound}

By following the derivation in [7], under the constraint $\mathbf{X} \in$ $\mathcal{X}$ the EPI lower bound reads

$$
\underline{C}_{\mathrm{EPI}}(N)=N \log \left(1+\frac{|\Lambda|^{\frac{2}{N}} \operatorname{Vol}(\mathcal{X})^{\frac{1}{N}}}{2 \pi e}\right),
$$

where the term $\operatorname{Vol}(\mathcal{X})$ is the $2 N$-dimensional volume of the set $\mathcal{X}$ in $\mathbb{R}^{2 N}$. Since $\mathcal{X}=\operatorname{Box}(\mathbf{a})$ can be interpreted as a Cartesian product, for the per-antenna constraint it holds

$$
\operatorname{Vol}(\mathcal{X})=\operatorname{Vol}\left(\mathcal{X}_{1} \times \cdots \times \mathcal{X}_{N}\right)=\prod_{k=1}^{N} \operatorname{Vol}\left(\mathcal{X}_{k}\right),
$$

where $\operatorname{Vol}\left(\mathcal{X}_{k}\right)=\operatorname{Vol}\left(\mathcal{B}_{2}(a)\right)=\pi a^{2}, \forall k$. Finally, from (10) and (11), we define the EPI lower bound, for the per-antenna constraint, as

$$
C_{\mathrm{MIMO}} \geq \underline{C}_{\mathrm{PA}}(N, a)=N \log \left(1+\frac{\prod_{k=1}^{N} \lambda_{k}^{\frac{2}{N}}}{2 e} a^{2}\right) .
$$

Nevertheless, this bound can be loose in presence of strong fading attenuation. Let us suppose that $\lambda_{N} \approx 0$ and $\lambda_{k}>$ $0, \forall k \neq N$. Since the contribution of each $k$ th subchannel, with $k \neq N$, is non-vanishing, it should be possible to reliably transmit at least on those subchannels. On the other hand, since $\lambda_{N} \approx 0$, it holds $\underline{C}_{\mathrm{PA}}(N, a) \approx 0$ for any practical SNR level. To improve tightness of the EPI lower bound, we introduce what we call piecewise-EPI lower bound. Contrary to the standard EPI lower bound, the piecewise-EPI bound selects only the reliable subchannels and excludes those with 


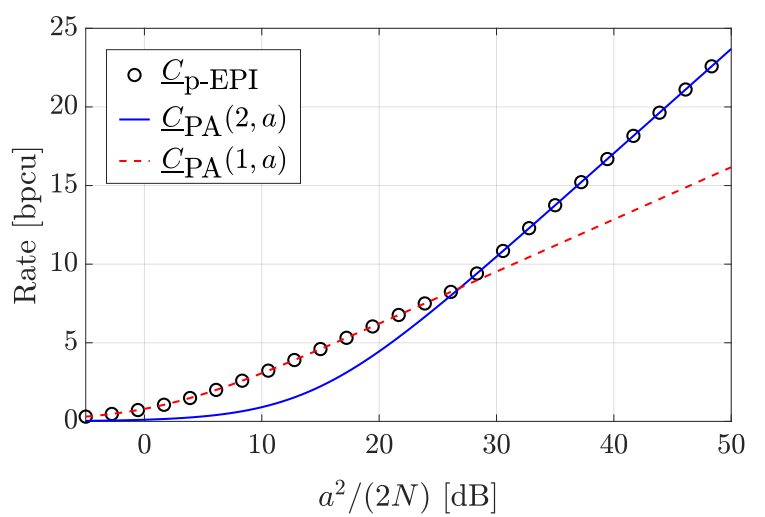

Figure 2. Comparison between the piecewise-EPI lower bound from (16) and the EPI lower bounds from (12).

vanishing $\lambda_{k}$, based on the SNR level. By data processing inequality, we have

$$
\begin{aligned}
C_{\mathrm{MIMO}} & =\max _{F_{\mathbf{X}_{1}^{N}} I}\left(\mathbf{X}_{1}^{N} ; \mathbf{Y}_{1}^{N}\right) \\
& \geq \max _{F_{\mathbf{X}_{1}^{k}}} I\left(\mathbf{X}_{1}^{k} ; \mathbf{Y}_{1}^{k}\right) \\
& \geq \underline{C}_{\mathrm{PA}}(k, a),
\end{aligned}
$$

for any positive integer $k \leq N$. Finally, the piecewise-EPI lower bound is

$$
\underline{C}_{\mathrm{p}-\mathrm{EPI}} \triangleq \max _{k}\left(\underline{C}_{\mathrm{EPI}}(k)\right)=\max _{k}\left(\underline{C}_{\mathrm{PA}}(k, a)\right) .
$$

In Fig. 2, we show how, for $N=2$ and $\boldsymbol{\lambda}=(1,0.05)^{T}$, having $\lambda_{N} \ll \lambda_{1}$ reduces the tightness of the bound at low-to-intermediate $\mathrm{SNR}$ values. In this $\mathrm{SNR}$ range, $\underline{C}_{\mathrm{PA}}(1, a)$ provides a tighter lower bound than $\underline{C}_{\mathrm{PA}}(2, a)$, by excluding the detrimental effect of processing the dimension with singular value $\lambda_{N}$. As the SNR increases, a larger number of subchannels become relevant for the final bound. Eventually, all of them are included and $\underline{C}_{\mathrm{p} \text {-EPI }}$ converges to $\underline{C}_{\mathrm{PA}}(N, a)$. Intuitively, the SNR becomes large enough to enable reliable communications also on those subchannels that suffer from stronger attenuation.

\section{Capacity Gap}

To assess the tightness of the bounds, let us define the capacity gap function $g(a)$ as

$$
g(a) \triangleq \bar{C}_{\mathrm{PA}}(a)-\underline{C}_{\mathrm{PA}}(N, a)
$$

We show that for increasing values of $a$ the gap goes to zero. Given that $\bar{C}_{k, \mathrm{PA}}(a)$ asymptotically approaches

$$
\lim _{a \rightarrow \infty} \bar{C}_{k, \mathrm{PA}}(a) \stackrel{(8)}{=} \lim _{a \rightarrow \infty} \log \left(\frac{\lambda_{k}^{2} a^{2}}{2 e}\right),
$$

it follows

$$
\begin{aligned}
& \lim _{a \rightarrow \infty} g(a) \\
& =\lim _{a \rightarrow \infty} \sum_{k=1}^{N} \log \left(\frac{\lambda_{k}^{2} a^{2}}{2 e}\right)-N \log \left(\frac{|\Lambda|^{\frac{2}{N}} a^{2}}{2 e}\right)=0 .
\end{aligned}
$$
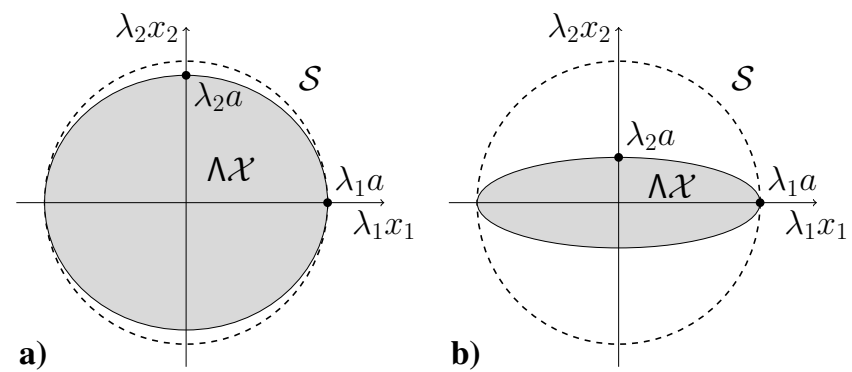

Figure 3. Set $\mathcal{S}=\mathcal{B}_{2}\left(\lambda_{1} a\right)$ and set $\Lambda \mathcal{X}$ for a $2 \times 2$ MIMO system with a) $\lambda_{1} \approx \lambda_{2}$ and with b) $\lambda_{1} \gg \lambda_{2}$.

\section{Total Amplitude Constraint}

The second case of practical interest is when a single amplifier feeds all the transmitting antennas, inducing a constraint on the norm of the input vector [8]. The constraint is $\widetilde{\mathbf{X}} \in \widetilde{\mathcal{X}}=\mathcal{B}_{2 N}(\widetilde{a}) \triangleq\{\widetilde{\mathbf{x}}:\|\widetilde{\mathbf{x}}\| \leq \widetilde{a}\}$, where $\mathcal{B}_{2 N}(\widetilde{a})$ is the $2 N$-dimensional ball in $\mathbb{R}^{2 N}$ centered in $\mathbf{0}_{2 N}$ and with radius $\widetilde{a}$. Since $\widetilde{\mathcal{X}}$ is a ball and $\mathrm{V}^{H}$ just performs a rotation, we have $\mathcal{X}=\lambda_{\max } \mathrm{V}^{H} \widetilde{\mathcal{X}}=\lambda_{\max } \widetilde{\mathcal{X}}$, and the constraint on $\widetilde{\mathrm{X}}$ is equivalent to $\mathbf{X} \in \mathcal{X}=\mathcal{B}_{2 N}(a)$ with $a=\lambda_{\max } \widetilde{a}$. As in the previous section, $\mathrm{SNR}=a^{2} /(2 N)$.

\section{A. Upper Bound}

We provide a capacity upper bound

$$
C_{\mathrm{MIMO}} \leq \max _{F_{\wedge \mathbf{X}}: \operatorname{supp}\left(F_{\wedge \mathbf{X}}\right) \subseteq \mathcal{S}} I(\Lambda \mathbf{X} ; \mathbf{Y})
$$

by using any enlarged set $\mathcal{S} \supseteq \wedge \mathcal{X}$. This allows us to choose a region $\mathcal{S}$ that makes the bound computation more tractable. Let us focus on the bound at high SNR. As shown in [9], as the SNR increases the channel capacity becomes proportional to $\log (\operatorname{Vol}(\Lambda \mathcal{X}))$. To make the bound tight, the volume of the set $\mathcal{S}$ should be as close as possible to that of $\wedge \mathcal{X}$. A simple choice would be $\mathcal{S}=\mathcal{B}_{2 N}\left(\lambda_{1} a\right)$. Let us consider, for instance, a $2 \times 2$ real MIMO system with $\boldsymbol{\lambda}=\left(\lambda_{1}, \lambda_{2}\right)^{T}$. If $\lambda_{1} \approx \lambda_{2}$, using $\mathcal{S}=\mathcal{B}_{2}\left(\lambda_{1} a\right)$ provides a good approximation to $\wedge \mathcal{X}$. On the contrary, whenever $\lambda_{1} \gg \lambda_{2}$, the ball is not a good choice. Both these two cases are shown in Fig. 3. The difference in volume between $\mathcal{S}$ and $\wedge \mathcal{X}$ becomes even more relevant as $N$ increases. Compared to $\mathcal{B}_{2 N}\left(\lambda_{1} a\right)$, a convenient choice of $\mathcal{S}$ is that of a Cartesian product of balls: it keeps the bound computation tractable, while reducing the volume difference between $\mathcal{S}$ and $\wedge \mathcal{X}$. To better approximate $\wedge \mathcal{X}$, we want to group, in the same ball, dimensions that correspond to similar values of $\lambda_{k}$ and to separate, in independent balls, dimensions with very different $\lambda_{k}$ 's. In this way, the balls constituting $\mathcal{S}$ divide the $N$ MIMO dimensions in subsets, decomposing $\mathbb{R}^{2 N}$ in the corresponding separate subspaces. Given a partition $p$, $N^{(p)}$ is the number of subsets in $p$, which corresponds to the number of balls in $\mathcal{S}$, and $n_{k}^{(p)}$ is the dimension of the $k$ th subset, corresponding to the dimension of the $k$ th ball. For $p$ to be a valid partition, it must hold $\sum_{k=1}^{N^{(p)}} n_{k}^{(p)}=N$. Let $\mathbf{X}_{k}^{(p)}, \mathbf{Y}_{k}^{(p)}$, and $\mathbf{Z}_{k}^{(p)}$ be the $k$ th subset of inputs, outputs, and noise, respectively. The projection of $\mathcal{X}$ onto the $k$ th 
subspace is $\mathcal{X}_{k}^{(p)}=\mathcal{B}_{2 n_{k}^{(p)}}(a)$, and $\Lambda_{k}^{(p)}$ is the corresponding $k$ th submatrix of $\Lambda$. Let $\lambda_{k}^{(p)}[j]$ be the element $(j, j)$ of the submatrix $\Lambda_{k}^{(p)}$, with $j=1, \ldots, n^{(p)}$ and $k=1, \ldots, N^{(p)}$, and let $\lambda_{k}^{(p)}=\lambda_{k}^{(p)}[1]=\max _{j}\left(\lambda_{k}^{(p)}[j]\right)$. The system model in (3) can be rewritten as

$$
\left[\begin{array}{c}
\mathbf{Y}_{1}^{(p)} \\
\vdots \\
\mathbf{Y}_{N^{(p)}}^{(p)}
\end{array}\right]=\left[\begin{array}{ccc}
\Lambda_{1}^{(p)} & \ldots & \mathrm{O}_{1 \times N^{(p)}} \\
\vdots & \ddots & \vdots \\
\mathbf{O}_{N^{(p)} \times 1} & \cdots & \Lambda_{N^{(p)}}^{(p)}
\end{array}\right]\left[\begin{array}{c}
\mathbf{X}_{1}^{(p)} \\
\vdots \\
\mathbf{X}_{N^{(p)}}^{(p)}
\end{array}\right]+\left[\begin{array}{c}
\mathbf{Z}_{1}^{(p)} \\
\vdots \\
\mathbf{Z}_{N^{(p)}}^{(p)}
\end{array}\right]
$$

with $\mathrm{O}_{k \times j}$ being the $n_{k}^{(p)} \times n_{j}^{(p)}$ matrix of zeros. Choosing $\mathcal{S}^{(p)}=\lambda_{1}^{(p)} \mathcal{X}_{1}^{(p)} \times \cdots \times \lambda_{N^{(p)}}^{(p)} \mathcal{X}_{N^{(p)}}^{(p)}$ makes each subspace of the MIMO system virtually independent and allows us to treat each subset separately. By following similar steps to those pointed out in (6) and (7) and because of the maximization over the enlarged set $\mathcal{S}^{(p)} \supseteq \wedge \mathcal{X}$, we have

$$
\begin{aligned}
C_{\text {MIMO }} & \leq \max _{F_{\Lambda \mathbf{X}}: \operatorname{supp}\left(F_{\Lambda \mathbf{X}}\right) \subseteq \mathcal{S}^{(p)}} \sum_{k=1}^{N^{(p)}} I\left(\Lambda_{k}^{(p)} \mathbf{X}_{k}^{(p)} ; \mathbf{Y}_{k}^{(p)}\right) \\
& \leq \sum_{k=1}^{N^{(p)}} \max I\left(\Lambda_{k}^{(p)} \mathbf{X}_{k}^{(p)} ; \mathbf{Y}_{k}^{(p)}\right) \leq \sum_{k=1}^{N^{(p)}} \bar{C}_{k}^{(p)},
\end{aligned}
$$

where the last maximization is over $F_{\Lambda_{k}^{(p)}} \mathbf{X}_{k}^{(p)}$ and such that $\operatorname{supp}\left(F_{\Lambda_{k}^{(p)} \mathbf{X}_{k}^{(p)}}\right) \subseteq \mathcal{S}_{k}^{(p)}=\lambda_{k}^{(p)} \mathcal{X}_{k}^{(p)}$. The subset $\mathcal{S}_{k}^{(p)}$ represents the projection of $\mathcal{S}^{(p)}$ onto the subspace spanned by $\mathbf{X}_{k}^{(p)}$, and $\bar{C}_{k}^{(p)}$ is any capacity upper bound for the $k$ th subchannel. Since the tightness of the bound depends on how closely $\operatorname{Vol}\left(\mathcal{S}^{(p)}\right)$ approximates $\operatorname{Vol}(\wedge \mathcal{X})$, the partition with minimum volume provides the tightest bound at high SNR. We denote the optimal partition by $o$, that is

$$
o=\arg \min _{p} \operatorname{Vol}\left(\mathcal{S}^{(p)}\right) .
$$

For an $N \times N$ complex MIMO system, the number of possible partitions is given by the Bell number $B_{2 N}=\sum_{k=0}^{2 N}\left\{\begin{array}{c}2 N \\ k\end{array}\right\}$, where $\left\{\begin{array}{c}i \\ j\end{array}\right\}$ represents the Stirling number of the second kind. In general, $B_{n}$ counts the number of ways in which a set with $n$ elements can be partitioned into disjoint, non-empty subsets. Bell numbers grow rapidly with $n$. Exploring all the possible partitions becomes computationally unfeasible as $n$ grows larger, unless the following property is exploited.

Lemma 1. Given two partitions $c$ and $d$ such that $n_{k}^{(d)}=$ $n_{k}^{(c)}, \forall k$, if c satisfies $\lambda_{k}^{(c)}[j] \geq \lambda_{k+1}^{(c)}[l], \forall k, j, l$, then $\operatorname{Vol}\left(\mathcal{S}^{(c)}\right) \leq \operatorname{Vol}\left(\mathcal{S}^{(d)}\right)$. Moreover, in the optimal partition, identical singular values are grouped together.

Proof: See [10].

Thanks to Lemma 1, the number of partitions to be explored drops to $2^{N-1}$. Even for small $N$, like $N=5$, we already get a relevant reduction, from $B_{10}=115975$ to $2^{4}$. It is now feasible to numerically determine the optimal partition $o$ by using (24). To compute the final bound we have to define $\bar{C}_{k}^{(o)}, \forall k=1, \ldots, N^{(o)}$. The McKellips bound, for an
$N$-dimensional MIMO system and the total amplitude (TA) constraint $\mathbf{X} \in \mathcal{B}_{N}(a)$, is derived in [5]. It reads

$$
\bar{C}_{\mathrm{McK}}\left(\mathcal{B}_{N}(a)\right)=\log \left(k_{N}(a)+\frac{\operatorname{Vol}\left(\mathcal{B}_{N}(a)\right)}{(2 \pi e)^{N / 2}}\right),
$$

where

$$
\begin{aligned}
& k_{N}(a)=\sum_{i=0}^{N-1}\left(\begin{array}{c}
N-1 \\
i
\end{array}\right) \frac{\Gamma\left(\frac{N-i}{2}\right)}{2^{i / 2} \Gamma(N / 2)} a^{i}, \\
& \operatorname{Vol}\left(\mathcal{B}_{N}(a)\right)=\frac{\pi^{\frac{N}{2}}}{\Gamma\left(\frac{N}{2}+1\right)} a^{N} .
\end{aligned}
$$

We apply the McKellips bound to each of the $N^{(o)}$ subchannels

$$
\bar{C}_{k, \mathrm{TA}}=\bar{C}_{k}^{(o)}=\bar{C}_{\mathrm{McK}}\left(\lambda_{k}^{(o)} \mathcal{X}_{k}^{(o)}\right),
$$

with $\lambda_{k}^{(o)} \mathcal{X}_{k}^{(o)}=\mathcal{B}_{2 n_{k}^{(o)}}\left(\lambda_{k}^{(o)} \cdot a\right)$. Finally, by summing up all the contributions, we get the final upper bound

$$
C_{\text {MIMO }} \leq \bar{C}_{\mathrm{TA}}=\sum_{k=1}^{N^{(o)}} \bar{C}_{k, \mathrm{TA}} .
$$

\section{B. Lower Bound}

The lower bound can be computed, again, via EPI by following [7]. Similarly to the per-antenna amplitude constraint, we can adopt the piecewise approach. Bound (16) still holds, as long as $\underline{C}_{\mathrm{PA}}(k, a)$ is replaced by

$$
\underline{C}_{\mathrm{TA}}(k, a)=k \log \left(1+\frac{\left[\prod_{i=1}^{k} \lambda_{i}^{\frac{2}{k}}\right] \operatorname{Vol}\left(\mathcal{X}_{1}^{k}\right)^{\frac{1}{k}}}{2 \pi e}\right),
$$

where $\mathcal{X}_{1}^{k}$ is the projection of $\mathcal{X}$ onto the first $k$ dimensions, with $k \leq N$, and

$$
\operatorname{Vol}\left(\mathcal{X}_{1}^{k}\right)=\frac{\pi^{k}}{\Gamma(k+1)} a^{2 k} .
$$

\section{Capacity Gap}

Let us consider the capacity gap function

$$
g(a)=\bar{C}_{\mathrm{TA}}(a)-\underline{C}_{\mathrm{TA}}(N, a) .
$$

We want to evaluate $\lim _{a \rightarrow \infty} g(a)$. Let us define $G(n)=$ $\pi^{\frac{n}{2}} / \Gamma\left(\frac{n}{2}+1\right)$, then the upper bound $\bar{C}_{\mathrm{TA}}(a)$ in the limit is given by

$$
\begin{aligned}
& \lim _{a \rightarrow \infty} \bar{C}_{\mathrm{TA}}(a) \\
& =\lim _{a \rightarrow \infty} \sum_{k=1}^{N^{(o)}} \bar{C}_{k, \mathrm{TA}} \\
& \stackrel{(28)}{=} \lim _{a \rightarrow \infty} \sum_{k=1}^{N^{(o)}} \log \left(\frac{\operatorname{Vol}\left(\lambda_{k}^{(o)} \mathcal{X}_{k}^{(o)}\right)}{(2 \pi e)^{n_{k}^{(o)}}}\right) \\
& =\lim _{a \rightarrow \infty} \sum_{k=1}^{N^{(o)}} \log \left(\frac{G\left(2 n_{k}^{(o)}\right)\left(\lambda_{k}^{(o)} a\right)^{2 n_{k}^{(o)}}}{(2 \pi e)^{n_{k}^{(o)}}}\right)
\end{aligned}
$$




$$
\begin{aligned}
= & \log \left(\prod_{k=1}^{N^{(o)}} G\left(2 n_{k}^{(o)}\right)\right)+\log \left(\prod_{k=1}^{N^{(o)}}\left(\lambda_{k}^{(o)}\right)^{2 n_{k}^{(o)}}\right) \\
& +\lim _{a \rightarrow \infty}\left[\log \left(a^{2 N}\right)\right]-\log \left((2 \pi e)^{N}\right) .
\end{aligned}
$$

As for the lower bound, we have

$$
\begin{aligned}
\lim _{a \rightarrow \infty} & C_{\mathrm{TA}}(N, a) \\
\stackrel{(30)}{=} & \lim _{a \rightarrow \infty} N \log \left(\frac{\left(G(2 N) a^{2 N}\right)^{\frac{1}{N}} \prod_{k=1}^{N} \lambda_{k}^{\frac{2}{N}}}{2 \pi e}\right) \\
= & \log (G(2 N))+\log \left(\prod_{k=1}^{N} \lambda_{k}^{2}\right) \\
& +\lim _{a \rightarrow \infty}\left[\log \left(a^{2 N}\right)\right]-N \log (2 \pi e) .
\end{aligned}
$$

Finally, the asymptotic gap is

$$
\begin{aligned}
g_{o} \triangleq \lim _{a \rightarrow \infty} g(a) & =\log \left(\prod_{k=1}^{N^{(o)}} G\left(2 n_{k}^{(o)}\right)\right)-\log (G(2 N)) \\
& +\log \left(\prod_{k=1}^{N^{(o)}}\left(\lambda_{k}^{(o)}\right)^{2 n_{k}^{(o)}}\right)-\log \left(\prod_{k=1}^{N} \lambda_{k}^{2}\right)
\end{aligned}
$$

Since the gap depends on the singular values and the associated optimal partition, it is difficult to formulate it with a compact expression. Let $g_{p}$ be the asymptotic gap for a partition $p$. Since $o$ minimizes the volume in $\bar{C}_{\mathrm{TA}}$ and $\underline{C}_{\mathrm{TA}}$ does not depend on $p$, we certainly have

$$
g_{o} \leq g_{p}, \forall p
$$

Often loose, but simple, upper bounds on $g_{o}$, can be computed by considering the two extreme cases $N^{(p)}=1$ and $N^{(p)}=$ $N$. For $N^{(p)}=1$ the only relevant singular value is $\lambda_{1}^{(p)}=\lambda_{1}$, which gives the gap

$$
g_{p, 1}=\log \left(\prod_{k=1}^{N}\left(\frac{\lambda_{1}}{\lambda_{k}}\right)^{2}\right) .
$$

Instead, when $N^{(p)}=N$, we have $\lambda_{k}^{(p)}=\lambda_{k}$, and the gap is

$$
g_{p, N}=\log \left(G(2)^{N}\right)-\log (G(2 N))=\log (N !) .
$$

Then, an upper bound on the gap $g_{o}$ is

$$
\begin{aligned}
g_{o} \leq \bar{g} & =\min \left\{g_{p, 1}, g_{p, N}\right\} \\
& =\min \left\{\log \left(\prod_{k=1}^{N}\left(\lambda_{1} / \lambda_{k}\right)^{2}\right), \log (N !)\right\} .
\end{aligned}
$$

Whenever $\lambda_{1} \approx \lambda_{N}$, the first term in (46) is the minimum, whereas if $\lambda_{N} \approx 0$ it is the second term that bounds (46). Notice that if $\lambda_{k}=\lambda_{j}, \forall k, j$, then $g_{p, 1}=0$ as shown in [5]. Despite its simplicity, $\bar{g}$ already improves upon the current literature. Let $g_{M}$ be the bound on the gap in [7, Theorem 6]. In the scenario here considered, $g_{M}$ is given by

$$
g_{M}=\log \left((2 \pi N)^{\frac{1}{2}} \prod_{k=1}^{N}\left(\lambda_{1} / \lambda_{k}\right)^{2}\right) .
$$

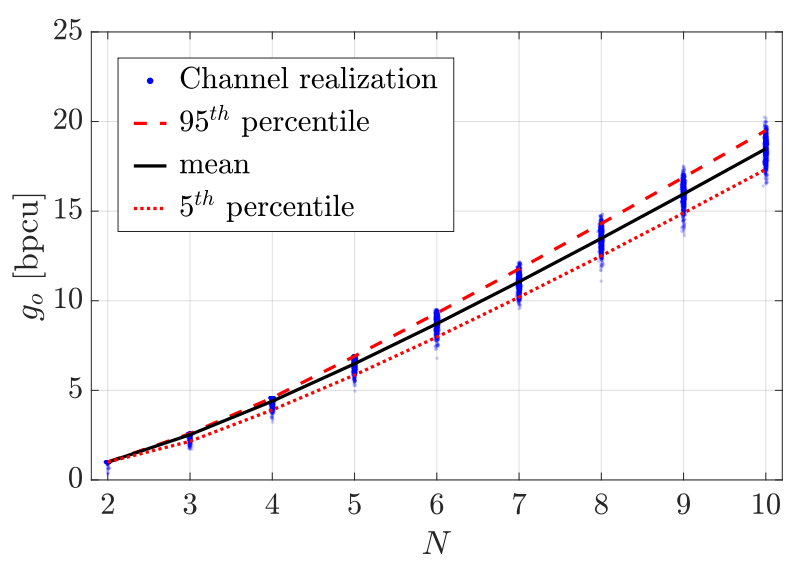

Figure 4. Scatter plot of $g_{o}$, from (41), versus $N$.

Since $g_{p, 1}$ in (45) is always smaller than $g_{M}$, we have

$$
g_{M}-\bar{g} \geq \frac{1}{2} \log (2 \pi N) .
$$

Moreover, let $g_{D}$ be the asymptotic gap between the upper bound in [7, Eq. (5)], adapted to our complex-valued model, and the EPI lower bound. We have

$$
g_{D}=\log (N !) \geq \bar{g}
$$

Finally, in Fig. 4, we show a scatter plot of $g_{o}$ versus $N$. For each channel realization, the entries of $\widetilde{H}$ are drawn as $\widetilde{H}_{i, j} \sim \mathcal{C N}(0,2), \forall i, j$. The residual gap gets worse for large $N$, because $\operatorname{Vol}\left(\mathcal{S}^{(o)}\right)$ approximates $\operatorname{Vol}(\Lambda \mathcal{X})$ less and less accurately. We conjecture that the lower bound is tight, while the inequality in (21) makes the upper bound loose. An upper bound proportional to $\log (\operatorname{Vol}(\wedge \mathcal{X}))$ would be significantly tighter, and the resulting gap would approach zero at high SNR, for any $N$.

\section{CONCLUSiON}

We evaluated capacity bounds for complex MIMO AWGN fading channels subject to input amplitude constraints. We focused on two practical cases, the first being a per-antenna constraints, while the second constraining the norm of the entire input vector. We introduced a refinement of the entropy power inequality lower bound, which improves its tightness at low-to-intermediate SNR in presence of strong fading attenuation. Finally, we evaluated the asymptotic gap between upper and lower bounds at high SNR. For the per-antenna constraint we showed that the gap approaches zero, and for the total amplitude constraint we obtained a smaller gap compared to those resulting from the best bounds in the literature.

\section{REFERENCES}

[1] J. G. Smith, "The information capacity of amplitude- and varianceconstrained scalar Gaussian channels," Information and Control, vol. 18, no. 3, pp. 203-219, April 1971.

[2] S. Shamai and I. Bar-David, "The capacity of average and peakpower-limited quadrature Gaussian channels," IEEE Transactions on Information Theory, vol. 41, no. 4, pp. 1060-1071, July 1995. 
[3] B. Rassouli and B. Clerckx, "On the capacity of vector Gaussian channels with bounded inputs," IEEE Transactions on Information Theory, vol. 62, no. 12, pp. 6884-6903, Dec 2016.

[4] A. L. McKellips, "Simple tight bounds on capacity for the peak-limited discrete-time channel," in International Symposium on Information Theory, 2004. ISIT 2004. Proceedings., June 2004, pp. 348-348.

[5] A. Thangaraj, G. Kramer, and G. Böcherer, "Capacity bounds for discrete-time, amplitude-constrained, additive white Gaussian noise channels," IEEE Transactions on Information Theory, vol. 63, no. 7 , pp. 4172-4182, July 2017.

[6] A. ElMoslimany and T. M. Duman, "On the capacity of multiple-antenna systems and parallel Gaussian channels with amplitude-limited inputs," IEEE Transactions on Communications, vol. 64, no. 7, pp. 2888-2899, July 2016.

[7] A. Dytso, M. Goldenbaum, S. Shamai, and H. V. Poor, "Upper and lower bounds on the capacity of amplitude-constrained MIMO channels," in GLOBECOM 2017 - 2017 IEEE Global Communications Conference, Dec 2017, pp. 1-6.

[8] S. Loyka, "The capacity of Gaussian MIMO channels under total and per-antenna power constraints," IEEE Transactions on Communications, vol. 65, no. 3, pp. 1035-1043, March 2017.

[9] V. Jog and V. Anantharam, "A geometric analysis of the AWGN channel with a $(\sigma, \rho)$-power constraint," IEEE Transactions on Information Theory, vol. 62, no. 8, pp. 4413-4438, Aug 2016.

[10] A. Favano, M. Ferrari, M. Magarini, and L. Barletta, "Supporting file for proof of Lemma 1," 2020. [Online]. Available: https: //www.dropbox.com/s/ov2bj12ytj0pt5k/SupportingFile.pdf 